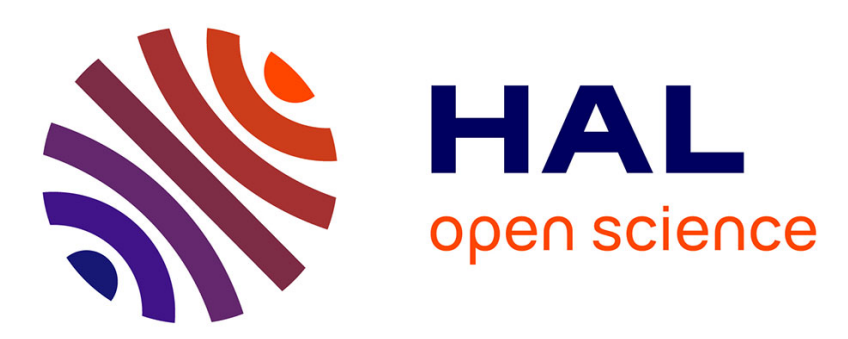

\title{
Quantum mechanical calculation of the rate constant for the reaction $\mathrm{H}+\mathrm{O}[$ sub 2] $\rightarrow \mathrm{OH}+\mathrm{O}$
}

\author{
Alexandra Viel, Claude Leforestier, William H. Miller
}

\section{To cite this version:}

Alexandra Viel, Claude Leforestier, William H. Miller. Quantum mechanical calculation of the rate constant for the reaction $\mathrm{H}+\mathrm{O}[$ sub 2] $\rightarrow \mathrm{OH}+\mathrm{O}$. Journal of Chemical Physics, 1998, 108 (9), pp.34893497. 10.1063/1.475776 . hal-01117492

\section{HAL Id: hal-01117492 \\ https://hal.science/hal-01117492}

Submitted on 10 Jul 2017

HAL is a multi-disciplinary open access archive for the deposit and dissemination of scientific research documents, whether they are published or not. The documents may come from teaching and research institutions in France or abroad, or from public or private research centers.
L'archive ouverte pluridisciplinaire HAL, est destinée au dépôt et à la diffusion de documents scientifiques de niveau recherche, publiés ou non, émanant des établissements d'enseignement et de recherche français ou étrangers, des laboratoires publics ou privés. 


\title{
Quantum mechanical calculation of the rate constant for the reaction $\mathrm{H}+\mathrm{O}_{2} \rightarrow \mathrm{OH}+\mathrm{O}$
}

\author{
Alexandra Viel and Claude Leforestier \\ Laboratoire Structure et Dynamique des Systèmes Moléculaires et Solides (UMR 5636), CC 014, \\ Université des Sciences et Techniques du Languedoc, 34095 Montpellier Cedex 05, France \\ William H. Miller \\ Department of Chemistry, University of California, and Chemical Sciences Division, Lawrence Berkeley \\ Laboratory, Berkeley, California 94720-1460
}

(Received 27 August 1997; accepted 26 November 1997)

\begin{abstract}
Quantum rate calculations for the $\mathrm{H}+\mathrm{O}_{2} \rightarrow \mathrm{HO}+\mathrm{O}$ combustion reaction, as well as for the reverse reaction, are reported. Using the DMBE IV potential energy surface, the cumulative reaction probability $N_{0}(E)$ has been directly computed for total angular momentum $J=0$, by means of the Seideman, Manthe and Miller Lanczos-based absorbing boundary condition method [J. Chem. Phys. 96, 4412 (1992); 99, 3411 (1993)]. Special attention has been paid to the definition of the molecular basis set, and to the sensitivity of the results to the absorbing potentials used in the asymptotic regions. The $N_{0}(E)$ results show very good overall agreement with the coupled channel calculations of Pack et al. [J. Chem. Phys. 102, 5998 (1995)], although the highly oscillatory behavior just above threshold renders such a comparison difficult in that energy range. The behavior of the $J \neq 0$ cumulative reaction probability has been estimated from calculations using the $J_{z}$-conserving approximation for $J$ values in the range 10-70. This allowed us to define which reference geometry should be used in the $J$-shifting approximation, in order to compute the cumulative reaction probability $N_{J}(E)$ for any $J$ value. By imposing conservation of the total energy within this approximation, the rate constants are shown to display better agreement with the experimental results. (C) 1998 American Institute of Physics. [S0021-9606(98)02609-9]
\end{abstract}

\section{INTRODUCTION}

The $\mathrm{HO}_{2}$ molecular system has received a great deal of theoretical interest recently, both as the intermediate complex for the combustion reaction ${ }^{1-12}$

$$
\mathrm{H}+\mathrm{O}_{2} \rightleftharpoons \mathrm{OH}+\mathrm{O},
$$

and as a realistic prototype for unimolecular dissociation ${ }^{13-18}$

$$
\left[\mathrm{HO}_{2}\right]^{*} \rightarrow \begin{cases}\mathrm{H}+\mathrm{O}_{2} & (\mathrm{I}) \\ \mathrm{OH}+\mathrm{O} & (\mathrm{II})\end{cases}
$$

These two aspects are closely related as the Cumulative Reaction Probability (CRP) $N(E)$ shows numerous resonance structures extending far above the reaction threshold., ${ }^{4,9}$ Those resonances can be expected to govern the unimolecular decomposition above the second threshold for dissociation (II). Such a behavior was found by Dobbyn et al. ${ }^{14,17}$ above the first threshold (I), with widths varying over five orders of magnitude.

In a preliminary study, ${ }^{5}$ we presented quantum mechanical calculations of the rate constant $k(T)$, using Seidemann and Miller's formulation ${ }^{19,20}$

$$
k(T)=\left[2 \pi \hbar Q_{R}(T)\right]^{-1} \int_{-\infty}^{+\infty} d E e^{-E / k T} N(E),
$$

which relies on a direct evaluation of the CRP $N(E)$. In order to compute $N(E)$, the Manthe and Miller ${ }^{21}$ expression

$$
N(E)=\operatorname{Tr}[\hat{P}(E)] \equiv \sum_{k} p_{k}(E)
$$

was used, where the reaction probability operator $\hat{P}$ is

$$
\hat{P}(E)=4 \hat{\epsilon}_{r}^{1 / 2} \hat{G}(E)^{\dagger} \hat{\epsilon}_{p} \hat{G}(E) \hat{\epsilon}_{r}^{1 / 2} .
$$

Here $\hat{G}(E)=(E+i \hat{\epsilon}-\hat{H})^{-1}$ is the Green's function as defined by Seideman and Miller, ${ }^{19}$ where $\hat{H}$ is the Hamiltonian operator and $\hat{\epsilon} \equiv \hat{\epsilon}_{r}+\hat{\epsilon}_{p}$ an absorbing potential which enforces outgoing wave boundary conditions, $\hat{\epsilon}_{r}\left(\hat{\epsilon}_{p}\right)$ being the part of the absorbing potential in the reactant (product) region. Manthe and Miller showed that a convenient way to evaluate the trace of $\hat{P}(E)$ in Eq. (3) is to determine its eigenvalues $\left\{p_{k}(E)\right\}$, the eigenreaction probabilities, whose values all lie between 0 and 1 . The eigenvalues of $\hat{P}$ were computed by the Lanczos procedure described in Ref. 21 . Thus if there are $n$ nonzero eigenvalues $\left\{p_{k}\right\}$ which contribute to the sum in Eq. (3), then $\sim n$ Lanczos iterations (plus maybe one or two) are required. This formulation was applied to the calculation of $N(E)$ for total angular momentum $J=0$, the higher $J$ values (up to $J=150$ ) being handled via the $J$-shifting approximation.

Recently, Germann and Miller ${ }^{12}$ extended this study to the case where both reaction and recombination $\mathrm{OH}+\mathrm{O} \rightarrow \mathrm{HO}_{2}$ are possible. In this formulation, the rate constants for reaction and recombination were respectively computed in terms of the flux correlation functions 


$$
\begin{aligned}
k_{p \leftarrow r}(T)=\left[Q_{r}(T)\right]^{-1} \int_{0}^{\infty} d t e^{-\omega t} C_{r p}(t), \\
k_{r e c o m b}(T)=\left[Q_{r}(T)\right]^{-1} \int_{0}^{\infty} d t\left(e^{-\omega t}-1\right)\left(C_{r r}(t)\right. \\
\left.-C_{r p}(t)\right),
\end{aligned}
$$

where $\omega$ is the collision frequency, and the correlations functions are defined from the flux operators $\hat{F}_{r}$ and $\hat{F}_{p}$

$$
\begin{aligned}
& C_{r r}(t)=\operatorname{tr}\left[e^{-\beta \hat{H} / 2} \hat{F}_{r} e^{-\beta \hat{H} / 2} e^{i \hat{H} t / \hbar} \hat{F}_{r} e^{-i \hat{H} t / \hbar}\right], \\
& C_{r p}(t)=\operatorname{tr}\left[e^{-\beta \hat{H} / 2} \hat{F}_{r} e^{-\beta \hat{H} / 2} e^{i \hat{H} t / \hbar} \hat{F}_{p} e^{-i \hat{H} t / \hbar}\right] .
\end{aligned}
$$

Due to the numerical complexity of the system, only the $J$ $=0$ rate constant was exactly computed, the contribution from higher $J$ values being accounted for by the $J$-shifting approximation. The results suggested that dampening of the forward and reverse reaction rates due to collisional recombination should become significant for pressures around $1000 \mathrm{~atm}$.

In the present work, using the Seidemann-Manthe-Miller formulation (Eqs. (2)-(4)), we reconsider our previous calculations of the rate constant $k(T)$ with special emphasis on the following points:

(1) as the $\mathrm{OH}+\mathrm{O}$ system displays long range forces, the reaction threshold is very sensitive to the actual definition of the basis set associated to the reaction coordinate. A larger grid was used in order to improve as far as possible the CRP behavior at threshold;

(2) the sensitivity of the CRP to the complex absorbing potentials $\epsilon_{r}$ and $\epsilon_{p}$ (Eq. (4)) used in the asymptotic regions has been checked, and quasi-independence has been achieved;

(3) the $J$-shifting approximation, ${ }^{22}$ which allows one to estimate the $J$ dependence of the CRP from a $J=0$ calculation, has been revisited. This approximation relies on defining a unique reference geometry at which the reaction is supposed to take place. As a consequence, asymptotic closed channels can contribute to the rate constant. We propose a modified version of this approximation which enforces conservation of the total energy.

The outline of this paper is as follows. In Sec. II, we first describe the $\mathrm{HO}_{2}$ molecular system, and present the $J=0$ calculations of the CRP $N(E)$. Section III explores the behavior of $N(E)$ for non zero $J$ values through the use of the $J_{z}$ conserving approximation, and compares it to the $J$-shifting results. In Sec. IV, we present the rate constant results as computed within the $J$-shifting approximation, and show how the modified version leads to better agreement with experimental results. Finally, Sec. V concludes.

\section{II. $J=0$ CALCULATIONS}

\section{A. Description of the system}

The $\mathrm{HO}_{2}$ molecular system is described in terms of the $\left(\mathbf{R}_{\mathrm{O}-\mathrm{O}}, \mathbf{r}_{\mathrm{H}, \mathrm{O}_{2}}\right)$ Jacobi coordinates. The resulting $J=0$ Body Fixed (BF) Hamiltonian $\mathbf{H}^{0}$ reads as
TABLE I. Definition of the two primary bases used in the calculations. These bases have been contracted as defined in Sec. II A.

\begin{tabular}{cccc}
\hline \hline & \multicolumn{4}{c}{$\begin{array}{c}\text { Representation } \\
\text { Reduced basis set }\end{array}$} & Dimension \\
\hline$r$ & Interval & Sine-DVR $\left\{\left|r_{n}\right\rangle, n=1, N_{r}\right\}$ & $N_{r}=70$ \\
$\theta$ & {$[0,7]$} & Odd Legendre polynomials $\{P /(\theta)\}$ & $N_{\ell}=48$ \\
$R$ & {$[2,10]$} & Sine-DVR $\left\{\left|R_{p}\right\rangle\right\}$ & $N_{R}=213$ \\
& \multicolumn{4}{c}{} \\
$r$ & {$[0,9]$} & Extended basis set \\
$\theta$ & {$[0, \pi]$} & Odd Legendre polynomials $\{P /(\theta)\}$ & $N_{r}=90$ \\
$R$ & {$[2,12]$} & Sine-DVR $\left\{\left|R_{p}\right\rangle\right\}$ & $N_{R}=48$ \\
& &
\end{tabular}

$$
\begin{aligned}
\mathbf{H}^{0}= & -\frac{\hbar^{2}}{2 \mu_{{\mathrm{H}, \mathrm{O}_{2}}_{2}}} \frac{\partial^{2}}{\partial r^{2}}-\frac{\hbar^{2}}{2 \mu_{\mathrm{O}_{2}}} \frac{\partial^{2}}{\partial R^{2}}-\hbar^{2}\left\{\frac{1}{2 \mu_{\mathrm{H}, \mathrm{O}_{2}} r^{2}}\right. \\
& \left.+\frac{1}{2 \mu_{\mathrm{O}_{2}} R^{2}}\right\} \frac{1}{\sin \theta} \frac{\partial}{\partial \theta} \sin \theta \frac{\partial}{\partial \theta}+V(r, R, \theta) .
\end{aligned}
$$

The potential used is the DMBE IV energy surface of Varandas et al. ${ }^{1,2}$ This system shows a deep well $(\simeq 2.4 \mathrm{eV})$ relative to the $\mathrm{H}+\mathrm{O}_{2}$ entrance valley, the $\mathrm{H}+\mathrm{O}_{2} \rightarrow \mathrm{O}+\mathrm{OH}$ reaction displaying an endothermicity of $0.714 \mathrm{eV}$ (including zero-point energies).

In order to represent the Hamiltonian operator of Eq. (9), we start from the following primary direct product basis set $\left\{\left|r_{n}\right\rangle\left|P_{\ell}\right\rangle\left|R_{q}\right\rangle\right\}$, where

- $\left\{\left|r_{n}\right\rangle, n=1, N_{r}\right\}$ and $\left\{\left|R_{q}\right\rangle, q=1, N_{R}\right\}$ are sine based discrete variable representation (DVR), ${ }^{23-25}$

- the $\left\{P /(\cos \theta), \ell=1,2 N_{\ell}-1\right\}$ Legendre basis set is restricted to odd functions because the ground state of the $\mathrm{O}-$ $\mathrm{O}-\mathrm{H}$ system is odd upon the interchange of the two oxygen atoms,

Two different $(r, R)$-grid definitions (reduced and extended), as given in Table I, have been used in the calculations.

Although very convenient, this primitive basis set is very large, and is associated to a broad spectral range which would limit its applicability in the subsequent $\mathscr{b}^{2}$ method under use. It is contracted in the following two steps:

(1) First, the $\left\{\left|R_{q}\right\rangle, q=1, N_{R}\right\}$ DVR is contracted by means of the HEG method ${ }^{26-28}$ in the following way. One defines an effective $V_{e f f}(R)$ potential as $V_{\text {eff }}(R)$ $=\min _{r, \theta} V(r, R, \theta)$. The $\mathbf{R}$ operator is then diagonalized in the truncated basis set built from the eigenfunctions of the $\mathbf{T}_{R}+V_{\text {eff }}(R)$ one dimensional Hamiltonian. The new $\left\{\left|\mathscr{R}_{p}\right\rangle\right\}$ DVR constitutes an adaptative grid with respect to the local de Broglie wavelength, as shown at the bottom of Fig. 1.

(2) The basis set associated to the two other Jacobi coordinates $(r, \theta)$ is defined via the sequential adiabatic reduction scheme of Bačić and Light. ${ }^{29,30}$ At each grid point $\mathscr{B}_{p}$, the $(r, \theta)$ Hamiltonian is diagonalized, and only the eigenfunctions $\left\{\Phi_{m}\left(r, \theta ; \mathscr{R}_{p}\right), m=1, M_{p}\right\}$ located below some threshold are retained. This threshold depends on the total energy $E$, and we have found that setting it 


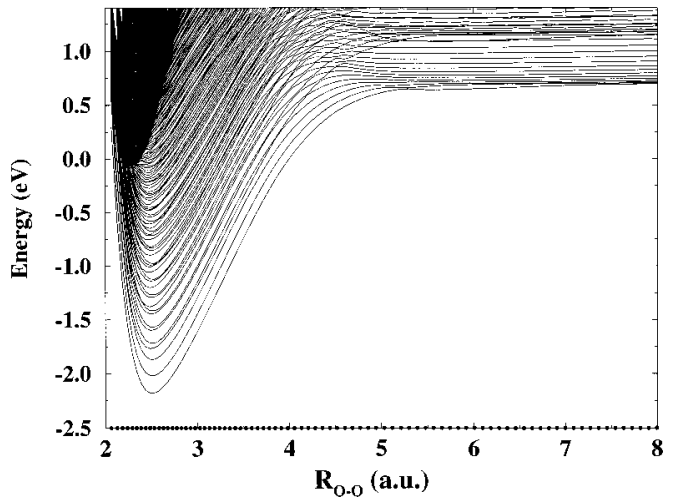

FIG. 1. Adiabatic energy curves with respect to the $R_{\mathrm{O}-\mathrm{O}}$ coordinate. These energies are referred to the zero-point energy of the $\mathrm{H}+\mathrm{O}_{2}$ reactants. The filled circles ( $)$ on the $R$ axis correspond to the HEG grid points.

0.3-0.4 eV above $E$ leads to converged calculations with respect to the basis set definition. For the $\mathrm{HO}_{2}$ system, using $R$ as the slow variable makes this scheme particularly efficient as the $(r, \theta)$ coordinates are associated to the motion of the light $\mathrm{H}$ atom relative to the heavy $\mathrm{O}-\mathrm{O}$ diatomic.

The basis set is finally written as $\left\{\left|\Phi_{m}\left(\mathscr{B}_{p}\right)\right\rangle\left|\mathscr{B}_{p}\right\rangle\right\}$ with a dimension ranging from 6731 to 11581 , depending on the threshold used. The corresponding adiabatic states are displayed in Fig. 1. One can note, for small separation of the two oxygen atoms, a very high density of states which are associated to the motion of the $\mathrm{H}$ atom in the $r$-box.

\section{B. Green's function calculation}

The basic step in the calculation of the CRP $N(E)$ is the application of the Green's function $\mathbf{G}(E)=(E+i \epsilon-\mathbf{H})^{-1}$ on some vector $|u\rangle$ :

$$
|g\rangle=\mathbf{G}(E)|u\rangle \text {. }
$$

Different schemes ${ }^{31-33}$ have been recently proposed, based on an expansion of $\mathbf{G}(E)$ in terms of polynomials of $\mathbf{H}$. In this work, we have solved the equivalent linear system

$$
(E+i \epsilon-\mathbf{H})|g\rangle=|u\rangle,
$$

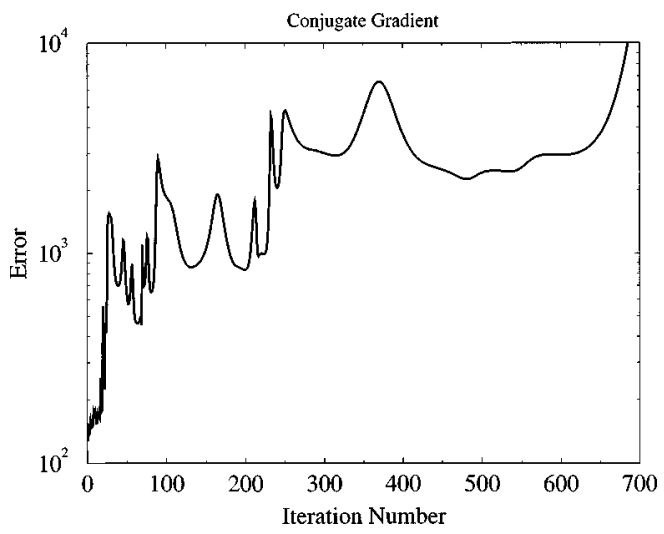

FIG. 2. Convergence of the Greens's function calculation, using the conjugate gradient algorithm, as a function of the number of iterations. The error represents the difference $\|(E+i \epsilon-\mathbf{H}) g-u\|$ in Eq. (11).

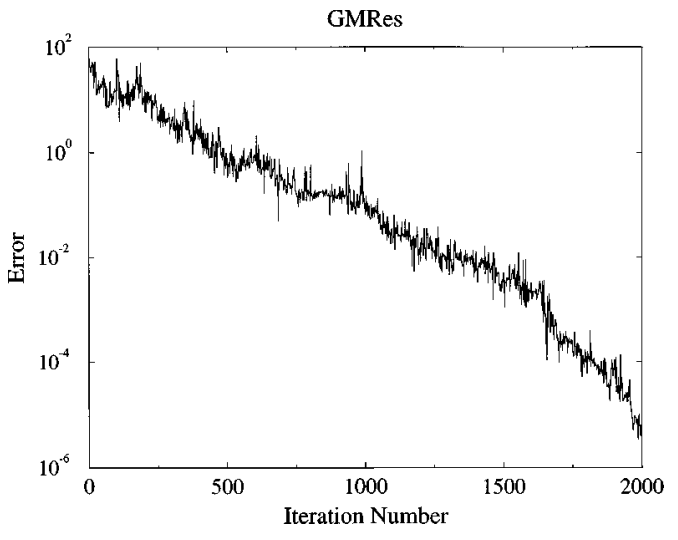

FIG. 3. Same as Fig. 2 for the GMRes algorithm.

testing two iterative (Conjugate Gradient, GMRes) and a direct (LU decomposition) approaches. The calculations reported below have been performed using a smaller contracted basis set of dimension 4338, as only the efficiency of the inversion scheme was to be tested.

\section{Conjugate gradient}

The Conjugate Gradient (CG) method $^{34}$ is based on a Lanczos scheme which brings the linear system to a tridiagonal form. Its main advantage is the reduced core memory needed, as only four vectors are required in order to advance the scheme. We present in Fig. 2 the convergence behavior of the CG resolution of Eq. (11). This graph displays the error $\|(E+i \epsilon-\mathbf{H}) g-u\|$ as a function of the number of iterations. It is clear that the scheme very quickly diverges.

\section{GMRes}

The GMRes algorithm ${ }^{35,36}$ consists in a reorthogonalized version of the conjugate gradient method. As a result, more core memory is required in order to store the successive iteration vectors. Figure 3 displays the convergence behavior as a function of the number of iterations. In this calculation, a tridiagonal preconditioning has been used. Although converging, the scheme necessitates a very large number of iterations, of the order of the basis set size.

\section{LU decomposition}

The LU decomposition scheme transforms the initial $(E+i \epsilon-\mathbf{H})$ matrix into the product $\underline{\underline{L}} \times \underline{\underline{U}}$ of two matrices, $\underline{\underline{L}}$ and $\underline{\underline{U}}$ being respectively of lower and upper triangular forms. This decomposition is performed only once for a given energy $E$, the Green's function being subsequently obtained by backward substitution.

Table II gives the computation times associated to these two steps, as well as those corresponding to the GMRes calculation, using the same initial vector $|u\rangle$. It can be seen that, due to the slow convergence of GMRes, the direct LU decomposition appears much more efficient, as the Green's function has to be evaluated only 5-20 times at each energy $E$ by backward substitution. 
TABLE II. Comparison of the GMRes and LU decomposition schemes for the Green's function calculation (Eq. (11). The CPU times are given for a Cray C 90 .

\begin{tabular}{ccc}
\hline \hline Scheme & CPU time $^{\mathrm{a}}$ & Core Memory $^{\mathrm{b}}$ \\
\hline GMRes & 682 & 18 \\
LU decomposition $^{\mathrm{c}}$ & 288 & 38 \\
LU Back transform & 0.2 & 38 \\
\hline \hline
\end{tabular}

${ }^{\mathrm{a} I n}$ seconds on Cray C90.

${ }^{\mathrm{b}}$ In Megawords.

${ }^{\mathrm{c}} \mathrm{NAG}$ Library.

\section{Test of the optical potential}

The CRP calculations rely on approximating the Green's function representation by means of optical potentials located in the asymptotic regions. We now explore the sensitivity of the CRP results with respect to the definition of these optical potentials. We have used an exponential potential,

$$
\epsilon_{\exp }(q)=C \exp \left(-\frac{\alpha\left(q_{\max }-q_{*}\right)}{q-q_{*}}\right),
$$

the $C$ and $\alpha$ parameters having been given by Vibok and Balint-Kurti ${ }^{37}$ in order to maximize its absorption with respect to the extension $L=q_{\max }-q_{*}$ and to the translational energy $E_{c o l}$. In Table III, we present test calculations of $N(E)$ with respect to the initial locations $R_{*}, r_{*}$, and the extensions $L_{R}$ and $L_{r}$ of the optical potentials. The extended basis definition has been used, in conjunction with a cut off energy of $1.23 \mathrm{eV}$ for the adiabatic basis set definition (see Sec. II A). These results correspond to a total energy $E=0.781 \mathrm{eV}$ with respect to the $\mathrm{H}+\mathrm{O}_{2}$ ground channel.

In these tests calculations, we successively varied the parameters of the optical potentials in the entrance $(r)$ and exit $(R)$ valleys. In both cases, the $N(E)$ value becomes stationary for the range value $L=2$ a.u., which will be used throughout the remaining of this paper.

\section{Test of the basis definition}

The extended basis (see Table I), which has been used in the previous tests, corresponds to a very large size at higher total energies. The reason is that the cut off value retained for defining the adiabatic states has to be increased with the energy. In another series of tests calculations, presented in

TABLE III. Variation of the $N(E)$ CRP with respect to parameters of the optical potential parameters. The total energy corresponds to $E=0.781 \mathrm{eV}$. Lengths are given in a.u.

\begin{tabular}{ccccc}
\hline \hline$r_{*}$ & $L_{r}$ & $R_{*}$ & $L_{R}$ & $N(E)$ \\
\hline 6.5 & 2.5 & 9.5 & 2.5 & 2.386 \\
7.0 & 2.0 &,, &, & 2.407 \\
7.5 & 1.5 &, &, & 2.336 \\
& & & & \\
7.0 & 2.0 & 9.0 & 3.0 & 2.350 \\
,, &,, & 9.5 & 2.5 & 2.407 \\
,, &, & 10.0 & 2.0 & 2.412 \\
,, &, & 10.5 & 1.5 & 2.301 \\
\hline \hline
\end{tabular}

TABLE IV. Comparison of $N(E)$ values with respect to the basis definition, as given in Table I.

\begin{tabular}{ccc}
\hline \hline Total energy $(\mathrm{eV})$ & Reduced basis & Extended basis \\
\hline 0.705 & 0.76 & $1.7(-3)$ \\
0.745 & 1.03 & 1.11 \\
0.781 & 2.40 & 2.41 \\
0.925 & 3.42 & 3.57 \\
1.025 & 5.03 & 4.91 \\
\hline \hline
\end{tabular}

Table IV, we compare the results coming from the two different bases for the total energies $E=0.705,0.745,0.781$, 0.925 and $1.025 \mathrm{eV}$.

These results show that the CRP value $N(E)$ depends only slightly on the basis definition except at very low energy. In particular, one can note the non zero CRP value obtained with the reduced basis at $E=0.705 \mathrm{eV}$, i.e., below the actual reaction threshold. The reason for that discrepancy stems from the presence of long range forces along the $\mathrm{OH} \cdots \mathrm{O}$ reaction coordinate. The reduced basis definition, which corresponds to an $R$-grid range, ${ }^{2,10}$ does not yet display a stabilized energy along the $\mathrm{O}-\mathrm{O}$ coordinate. The extended basis has thus been used for total energies below 0.92 $\mathrm{eV}$.

\section{E. $N_{J=0}(E)$ results}

We present in Fig. 4 the cumulative reaction probability $N_{J=0}(E)$ as a function of the collision energy, for the total
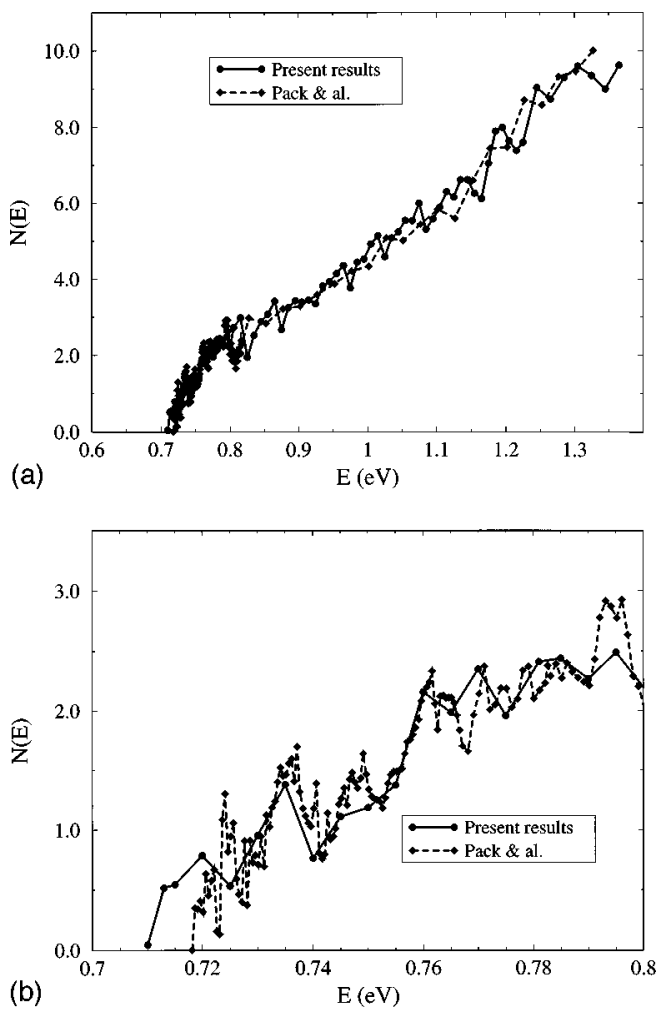

FIG. 4. The cumulative reaction probability $N_{J=0}(E)$ as a function of energy $E$ for total angular momentum $J=0$ : The full curve reports the present results, as obtained by Eq. (2), while the dashed curve corresponds to the coupled channel calculations of Pack and coworkers (Ref. 9): (a) the whole energy range studied; (b) blow up of the threshold region to show the resonance structure. 


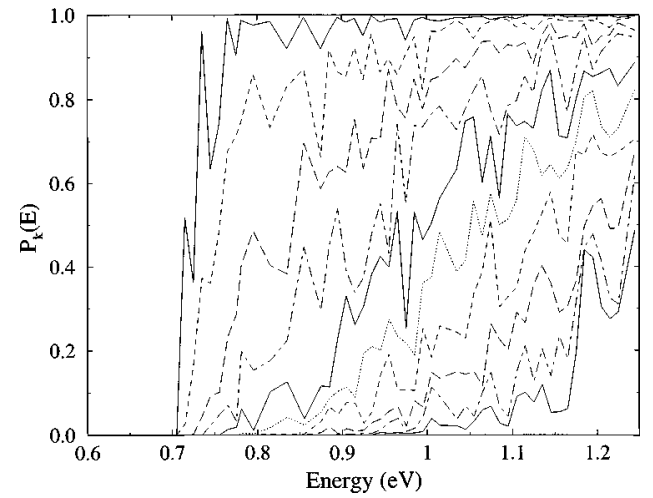

FIG. 5. Eigenreaction reaction probabilities $p_{k}(E)$-i.e., the eigenvalues of the matrix $\hat{P}(E)$ of Eq. (3), as a function of total energy.

angular momentum value $J=0$, altogether with the coupled channel calculations of Pack et al. ${ }^{9}$ The highly oscillatory behavior just above threshold renders the comparison difficult, but the overall agreement is very good. Figure 5 displays the corresponding individual eigenreaction probabilities $\left\{p_{k}(E)\right\}$ contributing to $N(E)$, as shown in Eq. (2). All these curves display resonance structures resulting from a collision complex caused by the deep well potential. The fact that the reaction probabilities do not rise promptly to unity with increasing energy is also a manifestation of dynamics that violates the basic tenet of transition state theory; i.e., all trajectories-using the language of classical mechanicswhich enter the interaction region do not proceed to products, but some turn around and become non reactive.

\section{III. $\boldsymbol{J} \neq \mathbf{0}$ APPROXIMATIONS}

Using the conservation of angular momentum $J$, the CRP $N(E)$ can be written as

$$
N(E)=\sum_{J}(2 J+1) N_{J}(E) .
$$

From this equation, the individual CRP's $N_{J}(E)$ should be calculated for each value of total angular momentum up to some $J_{\max }$ threshold. As will be shown in the next section, a $J_{\max }$ value in the range $60-150$ has to be considered, depending on the temperature. The $(J+1)$ dependence of the basis set size precludes any exact $N_{J \neq 0}$ calculation for the $\mathscr{L}^{2}$ method adopted here. Instead, we have considered two different approximations in order to estimate the $\mathrm{J}$ dependence of the CRP: the $J$-shifting approximation or the $J_{z}$ conserving formulation.

\section{A. The $J$-shifting approximation}

This approximation is essentially a molecular version $^{38,39}$ of the wave number approximation ${ }^{40}$ of atomic physics. It relies on the following assumptions:

(1) one neglects the coriolis coupling ( $K$, projection of $\mathbf{J}$ on the $\mathbf{z}$ BF axis, is a good quantum number) resulting in the relation

$$
N_{J}(E) \sim \sum_{K=-J}^{J} N_{J K}(E)
$$

TABLE V. Different complex geometries used in the $J$-shifting approximation. Distances are given in bohrs, and rotational constants in $\mathrm{cm}^{-1}$.

\begin{tabular}{llllcccc}
\hline \hline Complex & $R_{\mathrm{HO}}$ & $R_{\mathrm{OO}}$ & $\widehat{\mathrm{HOO}}$ & \multicolumn{1}{c}{$A$} & \multicolumn{1}{c}{$B$} & \multicolumn{1}{c}{$C$} & $\kappa$ \\
\hline $\mathrm{H} \cdots \mathrm{O}-\mathrm{O}$ & 4.0 & 2.29 & 117 & 6.14 & 1.13 & 0.96 & -0.631 \\
$\mathrm{H}-\mathrm{O}-\mathrm{O}$ & 1.85 & 2.54 & 104 & 20.25 & 1.10 & 1.04 & -0.891 \\
$\mathrm{H}-\mathrm{O} \cdots \mathrm{O}$ & 1.82 & 5.08 & 40 & 44.92 & 0.289 & 0.288 & -0.987 \\
\hline \hline
\end{tabular}

(2) the reaction takes place around some reference geometry $[A B C]^{\#}$, at which the rotational energy $E_{J K}^{\#}$ of the molecule is not available to overcome the transition state. The effective energy for the reaction to take place is therefore $E-E_{J K}^{\#}$, which leads to the further approximation

$$
N_{J K}^{\#}(E) \sim N_{J=0}\left(E-E_{J K}^{\#}\right) .
$$

For the case considered here, the first assumption should be verified as the reaction corresponds to the motion of a light atom with respect to the heavy oxygens. The occurrence of resonances in this reaction might however limit its applicability.

The second assumption is more intricate as it supposes a well defined transition state, where essentially the whole dynamics takes place. For a reaction with a deep well, this region is more dilute as it essentially spreads all over the interaction region. Resonances correspond to the system being trapped for a long time before dissociating in any of the two possible channels. In practice, two different "complexes" have been used, corresponding respectively to the bottom of the $\mathrm{H}-\mathrm{O}-\mathrm{O}$ well and to the $\mathrm{H}-\mathrm{O} \cdots \mathrm{O}$ transition state located in the exit valley. The corresponding geometries are given in Table $\mathrm{V}$, as well as the associated moments of inertia $A, B$ and $C$.

We present in Fig. 6 the $N_{J K=0}^{\#}(E)$ curves, as defined in Eq. (15), corresponding to these two different complexes, for values of $J$ in the range 10-70. The curves obtained from using the $\mathrm{H} \cdots \mathrm{O}-\mathrm{O}$ geometry (not represented here) are almost indistinguishable from those corresponding to the $\mathrm{H}-$ $\mathrm{O}-\mathrm{O}$ complex. To draw these curves, the rotational energy $E_{J K}^{\#}$ has been approximated by the symmetric top expression

$$
E_{J K}^{\#}=B^{\#} J(J+1)+\left(A^{\#}-B^{\#}\right) K^{2} .
$$

\section{B. $J_{z}$-conserving approximation}

In this formulation, ${ }^{41}$ one considers that $K$ is conserved during the reaction. As noted previously, the choice of $\mathrm{O}-\mathrm{O}$ as the $\mathbf{z} \mathrm{BF}$ axis tends to improve this approximation. Its net effect is to add the centrifugal term $\{J(J+1)$ $\left.-2 K^{2}\right\} /\left(2 \mu_{\mathrm{O}_{2}} r^{2}\right)$ to the Hamiltonian operator $\mathbf{H}^{0}$ of Eq. (9). These calculations require the same computational effort as those presented in the previous section for $J=0$.

We present in Fig. 7 the $N_{J K=0}^{(z)}(E)$ curves, obtained within this approximation, for values of $J$ in the range 1070. It should be noted that, as only the general behavior of these curves was of interest, only few total energies were sampled, resulting in apparent smoother curves than their $J$ $=0$ counterpart. Comparison of Figs. 6 and 7 shows that 


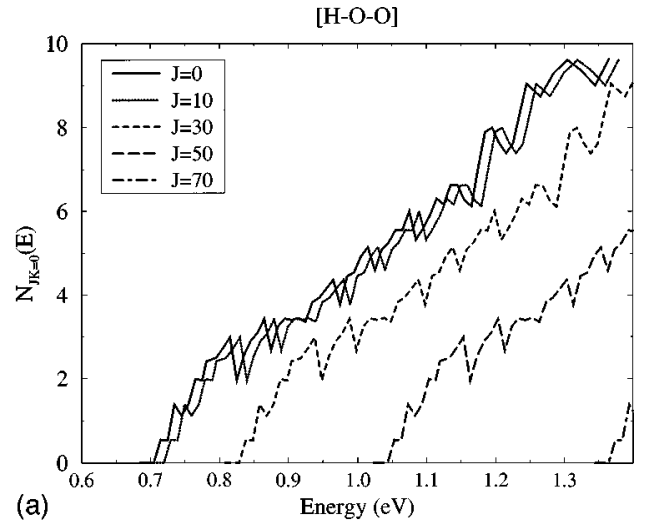

(a)

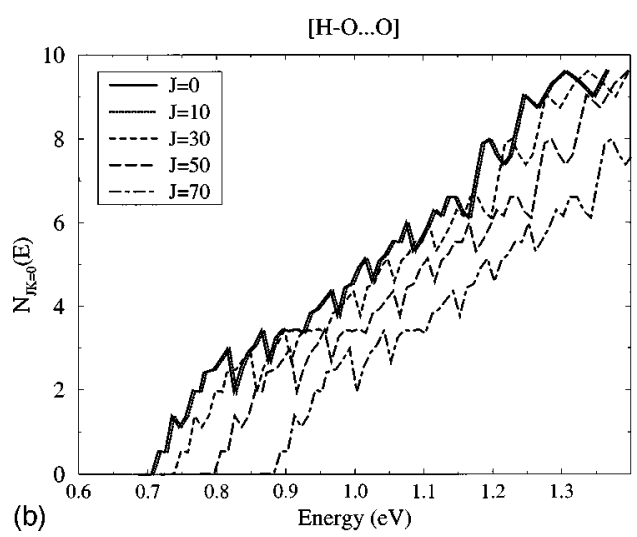

FIG. 6. The cumulative reaction probabilities $N_{J K=0}^{\#}(E)$, as obtained in the $J$-shifting approximation (Eq. (15)) for different values of total angular momentum $J$ : (a) $\mathrm{H}-\mathrm{O}-\mathrm{O}$ geometry; (b) $\mathrm{H}-\mathrm{O} \cdots \mathrm{O}$ geometry.

using the $\mathrm{H}-\mathrm{O} \cdots \mathrm{O}$ transition state geometry in the $J$-shifting approximation leads to a better agreement with the $J_{z}$ conserving results. If we consider these latter results as the best description available for such a complex system, they indicate that the $J$-shifting approximation should be used with the $\mathrm{H}-\mathrm{O} \cdots \mathrm{O}$ geometry as the reference one. This conclusion is in direct contradiction with the two previous studies realized on this system. ${ }^{5,12}$ Both of them made use of the H$\mathrm{O}-\mathrm{O}$ geometry as it leads to a better agreement with the experimental results. This contradiction is addressed in more detail in the next section.

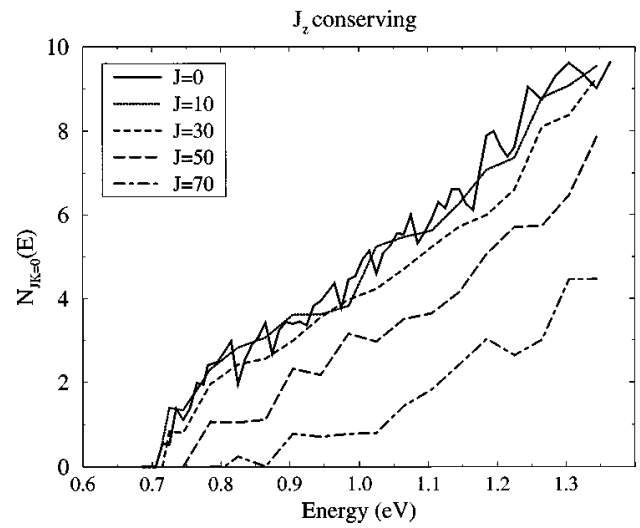

FIG. 7. The cumulative reaction probability $N_{J_{z} K=0}(E)$ as obtained in the $J_{z}$ conserving approximation for different values of total angular momentum $J$.

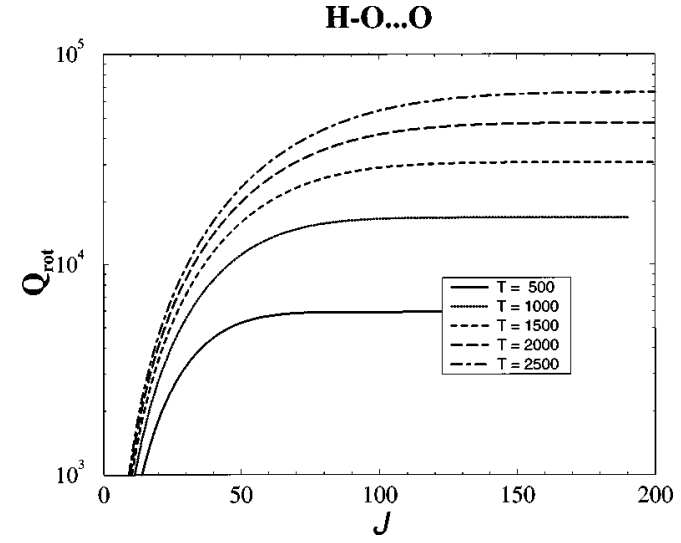

FIG. 8. Convergence of the rotational partition function $Q_{r o t}^{\#}(T)$ as a function of the maximum $J$ value entering Eq. (20), using the $\mathrm{H}-\mathrm{O} \cdots \mathrm{O}$ geometry as reference.

\section{RATE CONSTANT CALCULATIONS}

The canonical rate constant (Eq. (2)) displays the reactant partition function $Q_{R}(T)$ in front of its expression. This function is given by

$$
Q_{R}(T)=Q_{e l} \cdot Q_{v i b} \cdot Q_{\text {rot }} \cdot Q_{\text {trans }},
$$

where $Q_{v i b}$ and $Q_{r o t}$ are the standard vibrational and rotational partition functions for the diatomic $B C\left(\mathrm{O}_{2}\right.$ or $\left.\mathrm{OH}\right)$, and $Q_{\text {trans }}=\left(\mu_{A, B C} k T / 2 \pi \hbar^{2}\right)^{3 / 2}$ is the translational partition function (per unit volume) for relative motion of $A$ and $B C$.

The $Q_{e l}$ term deserves special attention as it takes into account the electronic degeneracy of the reactants: ${ }^{42}$

(1) the ground state reactants $\mathrm{H}\left({ }^{2} S\right)+\mathrm{O}_{2}\left({ }^{3} \Sigma_{g}^{-}\right)$have ${ }^{2,4} A^{\prime \prime}$ symmetry. As the $\mathrm{HO}_{2}$ surface considered here is of ${ }^{2} A^{\prime \prime}$ symmetry, ${ }^{43}$ the $Q_{e l}$ factor is $6 / 2$.

(2) For the reverse reaction $\mathrm{O}\left({ }^{3} P\right)+\mathrm{OH}\left({ }^{2} \Pi\right)$, the effective partition function ${ }^{44}$

$$
Q_{e l}=2\left\{\left[5+3 e^{-228 / T}+e^{-326 / T}\right]\left[2+2 e^{-205 / T}\right]\right\}^{-1}
$$

was used.

\section{A. Convergence tests}

Before presenting the rate constant calculations, we report some tests with respect to the maximum $J$ value and the total energy range required for convergence.

Using the $J$-shifting approximation, it is easily shown that the rate constant $k(T)$ can be expressed as

$$
k(T)=\left[2 \pi \hbar Q_{R}(T)\right]^{-1} Q_{r o t}^{\#}(T) \int_{-\infty}^{\infty} d E e^{-E / k T} N_{J=0}(E),
$$

where $Q_{r o t}^{\#}(T)$ is the rotational partition function of the supposed complex

$$
Q_{r o t}^{\#}(T)=\sum_{J=0}^{\infty}(2 J+1) \sum_{K=-J}^{J} e^{-E_{J K}^{\#} / k T} .
$$

Figure 8 displays the $J$-convergence of the rotational partition function as a function of the temperature, for the $\mathrm{H}-$ 


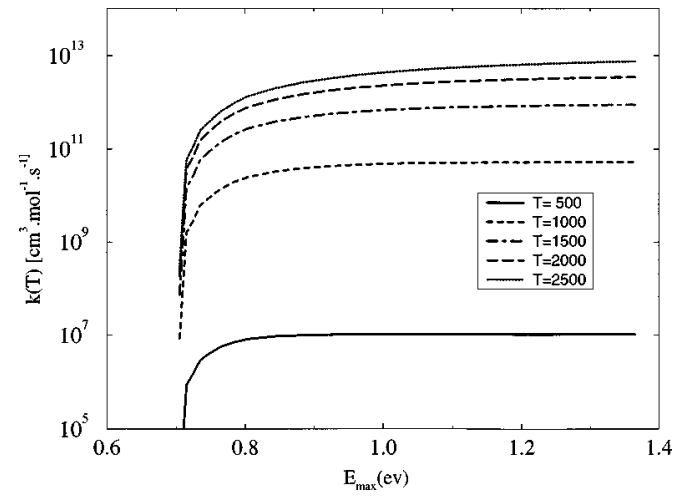

FIG. 9. Convergence of the rate constant $k(T)$ as a function of the upper integration limit $E_{\max }$ used in Eq. (19).

$\mathrm{O} \cdots \mathrm{O}$ geometry. This figure shows that very high $J$ values are required in the calculations, thereby mandating the use of the $J$-shifting approximation.

Our second convergence test concerns the upper integration limit, $E_{\max }$, to be used in the rate constant calculation (Eq. (19)). Figure 9 presents this rate constant for the O$\mathrm{O}-\mathrm{H}$ geometry as a function of $E_{\max }$, in the temperature interval 500-2500 K. It shows that the energy range sampled in our calculation, and shown in Fig. 4, allows in principle for a near convergence of the rate constant. In order to obtain this quantity at high temperatures, we have made use of a linear extrapolation of $N_{J=0}(E)$ beyond the last available data $E_{\text {sup }}=1.365 \mathrm{eV}$,

$$
N_{J=0}\left(E>E_{\text {sup }}\right)=N_{J=0}\left(E_{\text {sup }}\right) \times \frac{E-E_{\text {thr }}}{E_{\text {sup }}-E_{\text {thr }}},
$$

where $E_{t h r}$ corresponds to the threshold for reaction.

\section{B. Forward reaction}

Figure 10 shows the rate constant $k(T)$, as given by Eq. (2), in the temperature range $T=250-2500 \mathrm{~K}$, for the forward reaction. In this plot are reported the values obtained in

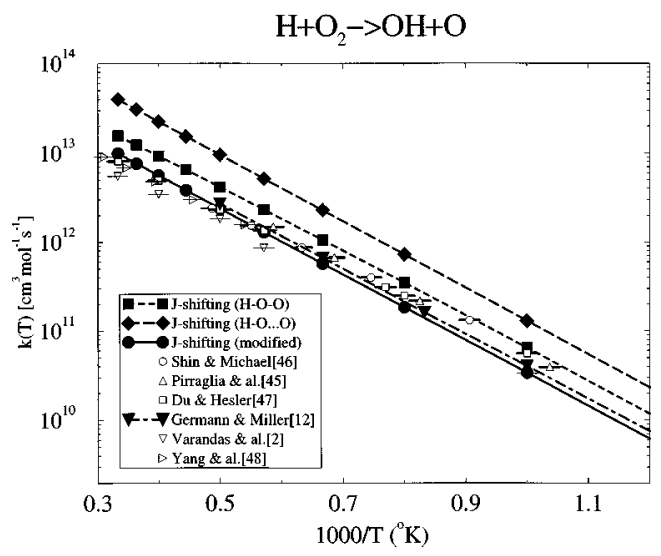

FIG. 10. Arrhenius plot of the forward reaction thermal rate constant $k(T)$, as given by Eq. (2), over the range $T=250-2500 \mathrm{~K}$. The dashed and long dashed curves correspond to the $J$-shifting approximation using the $\mathrm{H}-\mathrm{O}-\mathrm{O}$ and $\mathrm{H}-\mathrm{O} \cdots \mathrm{O}$ geometries respectively, the solid curve connecting the circles refers to its modified version, and the short-long dashed curve connecting the triangles recalls the results of Germann and Miller (Ref. 12). The quasiclassical results of Varandas et al. (Ref. 2) and the available experimental data (Refs. 45-48) are reported with their error bars.

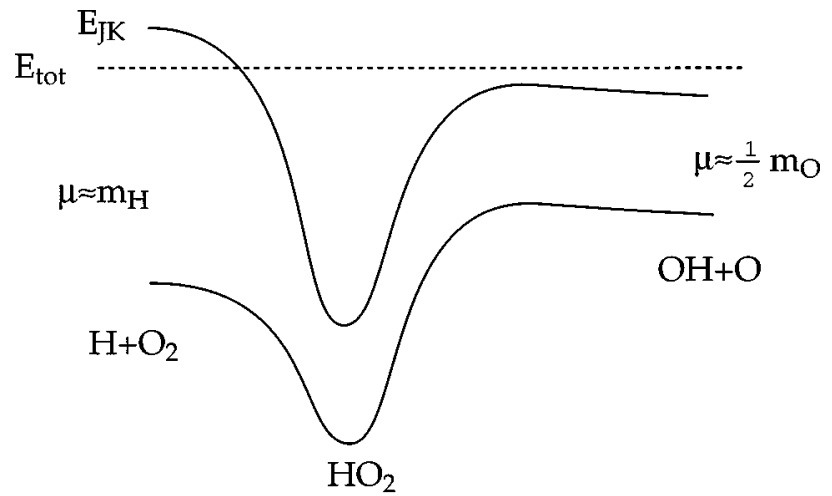

FIG. 11. Schematic representation of the variation of energy of a given $(J, K)$ rotational channel as a function of the geometry of the system.

the present calculations using the $J$-shifting approximation for the two reference geometries $(\mathrm{H}-\mathrm{O}-\mathrm{O}$ and $\mathrm{H}-\mathrm{O} \cdots \mathrm{O})$, those computed by German and Miller ${ }^{12}$ from the flux correlation functions and based on the $J$-shifting approximation (using the $\mathrm{H}-\mathrm{O}-\mathrm{O}$ geometry), the quasi classical results of Varandas and coworkers, ${ }^{2}$ as well as the experimental data available. ${ }^{45-48}$ The striking fact concerns the better agreement between the experimental data and the calculations associated with the $\mathrm{H}-\mathrm{O}-\mathrm{O}$ reference geometry, although the $J_{z}$-conserving results revealed that the $\mathrm{H}-\mathrm{O} \cdots \mathrm{O}$ geometry lead to a better description (see Figs. 6 and 7). It thus appears that such an agreement is fortuitous, and that the $J$-shifting approximation should be revisited.

The cumulative reaction probability $N_{J}(E)$ is defined as a sum over all the open reactive and products channels

$$
N_{J}(E)=\sum_{n_{R}, n_{P}}^{\text {open }}\left|S_{n_{P} \leftarrow n_{R}}(E)\right|^{2} .
$$

As formulated in Eq. (15), the $J$-shifting approximation considers a $(J, K)$ rotational channel to be open at total energy $E$ at the sole condition that $E-E_{J K}^{\#}$ be located above the reaction threshold $E_{t h r}, E_{J K}^{\#}$ corresponding to the rotational energy of the reference complex. Clearly, this allows for closed $(J, K)$ rotational channels to contribute to $N(E)$ in the situation where the entrance and exit channels are associated to different reduced masses, as shown in Fig. 11. This is the case for the reaction under study with approximate reduced masses of $m_{H}$ and $m_{\mathrm{O}} / 2$ in the entrance and exit valleys respectively.

We have thus modified the $J$-shifting approximation in order to enforce that only the open channels are taken into account. Assuming $K$ to be a good quantum number, a given $(J, K)$ rotational channel was considered as open at total energy $E$ if the two following conditions were met

$$
\begin{aligned}
& E-E_{J K}^{\# I n} \geqslant E_{T S}^{\# I n} \\
& E-E_{J K}^{\# O u t} \geqslant E_{T S}^{\# O u t},
\end{aligned}
$$

where $E_{J K}^{\#}$ and $E_{T S}^{\#}$ refer respectively to the rotational energy (Eq. (16)) and the transition state energy (including the zero point) of the complex. It should be noted that the assumption of $K$ being a good quantum number is now invoked in the whole interaction region; 


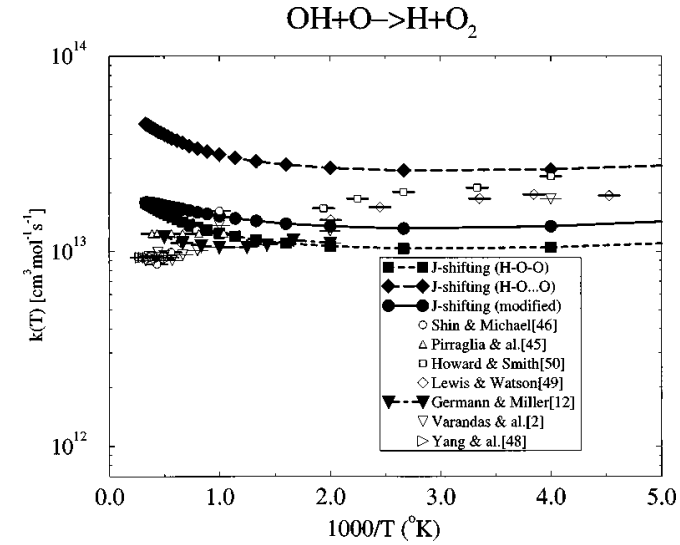

FIG. 12. Same as Fig. 10 for the reverse reaction.

$$
[\mathrm{H} \cdots \mathrm{O}-\mathrm{O}]^{\#} \leftrightarrow \mathrm{H}-\mathrm{O}-\mathrm{O} \leftrightarrow[\mathrm{H}-\mathrm{O} \cdots \mathrm{O}]^{\#},
$$

whereas in the usual $J$-shifting approximation, this assumption is only made in the vicinity of the transition state. Although the rotational constants change significantly in that region (see Table $\mathrm{V}$ ), the values of these constants are essentially determined from the $\mathrm{O}-\mathrm{O}$ distance. This new assumption is henceforth tantamount to postulating an adiabatic motion of the heavy $\mathrm{O}-\mathrm{O}$ bond in the field created by the light $\mathrm{H}$ atom.

Using $\mathrm{H}-\mathrm{O} \cdots \mathrm{O}$ as the reference geometry in the $J$-shifting approximation (Eq. (15)), we present in Fig. 10 the rate constant as computed from this modified formulation. It appears that the new curve is much closer to the experimental values. The agreement is especially good in the high energy regime for which the case of a $(J, K)$ rotational channel being closed in the entrance channel is much more probable due to the smaller reduced mass.

\section{Reverse reaction}

We present in Fig. 12 the rate constant $k(T)$ for the reverse reaction in the temperature range $200-2500 \mathrm{~K}$, as well as the experimental measurements available. ${ }^{49,50,45,46}$ One can first note that the uncorrected $J$-shifting approximation based on the $\mathrm{H}-\mathrm{O} \cdots \mathrm{O}$ reference geometry leads to a better agreement than $\mathrm{H}-\mathrm{O}-\mathrm{O}$ at low temperatures. This corroborates our previous conclusions drawn from using the $J_{z}$-conserving approximation, as $N(E)$ is the same for both the forward and reverse reactions. At higher temperatures, our corrected $J$-shifting calculations reproduce the quasiindependence of the rate constant with temperature observed experimentally.

\section{DISCUSSION}

We have reported new calculations of the rate constants for the forward and reverse $\mathrm{H}+\mathrm{O}_{2} \rightleftharpoons \mathrm{OH}+\mathrm{O}$ combustion reactions, in the absence of recombination. The method consisted in a direct evaluation of the cumulative reaction probability, using Seideman-Manthe-Miller formulation. This method appears especially efficient for endothermic reactions as the number of eigenreaction probabilities $p_{k}(E)$
(Eqs. (3)-(4)) to be iteratively determined is comparable to the number of open products asymptotic channels.

The Green's function $\mathbf{G}(E)$ has been evaluated by a direct $L U$ decomposition of the energy shifted Hamiltonian matrix. Such a scheme is very efficiently handled on a vector computer (sustained speed of 800 MFLOPS on a Cray C90), but it requires the whole matrix to be stored in core memory. To this end, the molecular basis set has been contracted as much as possible using the successive adiabatic reduction method of Bačic and Light. ${ }^{29,30}$ For such complex systems, recently proposed expansions of $\mathbf{G}(E)$ in terms of polynomials of $\mathbf{H}^{31-33}$ might be preferable as they rely on a direct evaluation of the Hamiltonian operator on some vector, thus avoiding the large core memory requirement. It must however be kept in mind that these schemes are generally used in some primitive basis set, corresponding to no or very little preconditioning. As a consequence, the subsequent iterative scheme is more difficult to converge due to the broader energy spectrum of the Hamiltonian in such a primitive basis set.

Special attention has been paid to the definition of the optical potentials used in the asymptotic regions, in order to achieve quasi-independence of the CRP value with respect to their parameters. The convergence of our results has been checked by comparison with the coupled channel calculations of Pack and coworkers. 9

As only exact calculations are feasible for total angular momentum $J=0$, the $J$-dependence of the CRP has been computed within the $J$-shifting approximation. This approximation relies on some reference geometry at which the reaction is supposed to take place. Two different geometries can be used as such a reference, namely the bottom of the well ( $\mathrm{H}-\mathrm{O}-\mathrm{O})$ or the transition state located in the product valley $(\mathrm{H}-\mathrm{O} \cdots \mathrm{O})$, leading to rate constant values differing by a factor of 2 over the whole temperature range. In order to estimate the correct $J$-dependence of the CRP, we employed the $J_{z}$ conserving approximation $(K=0)$ for values of $J$ in the range $10-70$. Such an approximation is particularly justified in the present case, as the reaction can be viewed as the motion of a light atom around a breaking heavy diatomic molecule. This study revealed that the transition state $\mathrm{H}-$ $\mathrm{O} \cdots \mathrm{O}$ geometry should be used, whereas the $J$-shifting results based on the $\mathrm{H}-\mathrm{O}-\mathrm{O}$ complex geometry lead to better agreement with the experimental data. This apparent paradox was solved by revisiting the $J$-shifting approximation, and imposing to consider only the rotational $(J, K)$ channels which are open with respect to both reactants and products. This modification is of particular importance for a $\mathrm{L}+\mathrm{HH}$ $\rightarrow L H+H$ type reaction, which displays very different reduced masses in the entrance and exit valleys.

\section{ACKNOWLEDGMENT}

This work was supported by a grant of computer time from the Institut du Développement et des Ressources en Informatique Scientifique (IDRIS), and by the Director, Office of Energy Research, Office of Basic Energy Sciences, Chemical Sciences Division, of the U.S. Department of Energy under Contract No. DE-AC03-76SF00098 and also by the National Science Foundation under Grant No. CHE9422559. 
${ }^{1}$ M. R. Pastrana, L. A. M. Quintales, J. Brandao, and A. J. C. Varandas, J. Phys. Chem. 94, 8073 (1990).

${ }^{2}$ A. J. C. Varandas, J. Brandao, and M. R. Pastrana, J. Chem. Phys. 96, 5137 (1992)

${ }^{3}$ A. J. C. Varandas, J. Chem. Phys. 99, 1076 (1993).

${ }^{4}$ R. Pack, E. Butcher, and G. Parker, J. Chem. Phys. 99, 9310 (1993).

${ }^{5}$ C. Leforestier and W. H. Miller, J. Chem. Phys. 100, 733 (1900).

${ }^{6}$ D. Zhang and J. Zhang, J. Chem. Phys. 101, 3671 (1994).

${ }^{7}$ V. Barclay et al., J. Chem. Phys. 103, 3864 (1995).

${ }^{8}$ B. Kendrick and R. Pack, J. Chem. Phys. 102, 1994 (1995).

${ }^{9}$ R. Pack, E. Butcher, and G. Parker, J. Chem. Phys. 102, 5998 (1995).

${ }^{10}$ A. Varandas, Chem. Phys. Lett. 235, 111 (1995).

${ }^{11}$ B. Kendrick and R. Pack, J. Chem. Phys. 104, 7475, 7502 (1996); 106, 3519 (1997)

${ }^{12}$ T. Germann and W. Miller, J. Phys. Chem. A. 101, 6358 (1997).

${ }^{13}$ D. Chapman, J. Bowman, and B. Gazdy, J. Chem. Phys. 96, 1919 (1992).

${ }^{14}$ A. J. Dobbyn et al., J. Chem. Phys. 102, 5867 (1995).

${ }^{15}$ B. Kendrick and R. Pack, Chem. Phys. Lett. 235, 291 (1995).

${ }^{16}$ V. Mandelshtam, T. P. Grozdanov, and H. Taylor, J. Chem. Phys. 103, 10074 (1995).

${ }^{17}$ A. Dobbyn, M. Stumpf, H.-M. Keller, and R. Schinke, J. Chem. Phys. 104, 8357 (1996).

${ }^{18}$ A. Varandas, J. Bowman, and B. Gazdy, Chem. Phys. Lett. 235, 405 (1995).

${ }^{19}$ T. Seideman and W. H. Miller, J. Chem. Phys. 96, 4412 (1992).

${ }^{20}$ T. Seideman and W. H. Miller, J. Chem. Phys. 97, 2499 (1992).

${ }^{21}$ U. Manthe and W. H. Miller, J. Chem. Phys. 99, 3411 (1993).

${ }^{22}$ J. W. Bowman, J. Phys. Chem. 95, 4960 (1991).

${ }^{23}$ J. C. Light, I. P. Hamilton, and J. V. Lill, J. Chem. Phys. 82, 1400 (1985).

${ }^{24}$ C. Leforestier, J. Chem. Phys. 94, 6388 (1991).

${ }^{25}$ D. T. Colbert and W. H. Miller, J. Chem. Phys. 96, 1982 (1992).

${ }^{26}$ D. O. Harris, G. G. Engerholm, and W. D. Gwinn, J. Chem. Phys. 43, 1515 (1965).

${ }^{27}$ C. Leforestier, in 4th Topsoe Summer School on Time Dependent Methods in Quantum Mechanics (G. D. Billing, Copenhagen, 1991).

${ }^{28}$ J. Echave and D. C. Clary, Chem. Phys. Lett. 190, 225 (1992).

${ }^{29}$ Z. Bačić and J. C. Light, J. Chem. Phys. 85, 4594 (1986).

${ }^{30}$ Z. Bačić and J. C. Light, J. Chem. Phys. 87, 4008 (1987).

31 S. M. Auerbach and W. H. Miller, J. Chem. Phys. 100, 1103 (1994).

${ }^{32}$ W. Zhu et al., Chem. Phys. Lett. 217, 74 (1994).

${ }^{33}$ T. P. Grozdanov, V. A. Mandelshtam, and H. S. Taylor, J. Chem. Phys. 103, 23 (1995).

${ }^{34}$ E. Strang, Numerical Analysis (Wellesey, Cambridge, 1986).

${ }^{35}$ Y. Saad and M. H. Schultz SIAM (Soc. Ind. Appl. Math.) J. Sci. Stat. Comput. 7, 856 (1986).

${ }^{36}$ M. S. Reeves, D. C. Chatfield, and D. G. Truhlar, J. Chem. Phys. 99, 2739 (1993).

${ }^{37}$ A. Vibok and G. G. Balint-Kurti, J. Phys. Chem. 96, 8712 (1992).

${ }^{38}$ J. M. Bowman, Adv. Chem. Phys. 61, 115 (1985).

${ }^{39}$ S. L. Mielke, G. C. Lynch, D. G. Truhlar, and D. W. Schwenke, Chem. Phys. Lett. 216, 441 (1993).

${ }^{40}$ K. Takayanagi, Prog. Theor. Phys. 8, 497 (1952).

${ }^{41}$ R. E. Wyatt, in Atom-Molecule Collision Theory, edited by R. B. Bernstein (Plenum, New York, 1979), p. 477.

${ }^{42}$ D. Truhlar, J. Chem. Phys. 56, 3189 (1972).

${ }^{43}$ S. P. Walch, C. Rohlfing, C. F. Melius, and C. W. Bauschlicher, J. Chem. Phys. 88, 6273 (1988).

${ }^{44}$ M. M. Graff and A. F. Wagner, J. Chem. Phys. 92, 2423 (1990).

${ }^{45}$ A. N. Pirraglia, J. V. Michael, J. W. Sutherland, and R. B. Klemm, J. Phys. Chem. 93, 282 (1989).

${ }^{46}$ K. S. Shin and J. V. Michael, J. Chem. Phys. 95, 262 (1991).

${ }^{47}$ H. Du and J. P. Hessler, J. Chem. Phys. 96, 1077 (1992).

${ }^{48}$ H. Yang, W. Gardiner, K. Shin, and N. Fuji, J. Chem. Phys. 101, 3671 (1994).

${ }^{49}$ R. S. Lewis and R. T. Watson, J. Phys. Chem. 84, 3495 (1980)

${ }^{50}$ M. J. Howard and I. W. M. Smith, J. Chem. Soc. Faraday Trans. 77, 997 (1981). 